\title{
Three Cases of Defect of the Left Common Carotid Artery in the Rabbit
}

\author{
By
Hitoshi OKUDA, Shigenori OKADA, Isumi TODA, Shigeki FUJIWARA, Hiromasa INOUE and Yoshikuni OHTA \\ Department of Anatomy, Osaka Dental University \\ 1-47, Kyobashi, Higashi-ku, Osaka 540, Japan \\ (Director: Prof. Y. Ohta) \\ (with 1 table and 11 figures in 3 plates)
}

-Received for Publication, March 7, 1988-

Key words: Common carotid artery/abnormalities, Rabbit.

Summary: In three individuals (\#1, \#2, and \#3) among 906 adult rabbits, in which vascular casts and dissection specimens of the whole vascular system prepared by acryl plastic injection were examined, the left common carotid artery was found to be defective.

The aortic arch in each of these three cases was located at a normal height. The branches of the arch in usual cases in the rabbit were the brachiocephalic trunk and the left subclavian artery. This trunk trifurcated into the left and right common carotid arteries and the right subclavian artery, as seen in Type B of man (Adachi 1928). However, the left common carotid was not observed in each of the examples reported here. In the third example (\#3), which was a dissection specimen, a remnant consisting of a connective tissue cord, instead of the left common carotid, was found. The right common carotid and right external carotid arteries were thicker than those observed in usual cases without any variations in their course and ramifications. The lingual artery in each of the three examples was rather thick and anastomosed strongly via a thick, hyoid branch with its opposite fellow. The left common carotid appeared as a fine vessel passing caudally from the bifurcation of the common carotid artery and became a fine cord of connective tissue which joined the brachiocephalic trunk. The bilateral lingual arteries were thick and anastomosed with each other. The hyoid branch of the lingual artery of the rabbit was always thick. Through this anastomosis, the blood supply to the left half of the head was performed by ramifications of the right external carotid. It can thus be said that, prior to closure of the left common carotid, the above-mentioned anastomosis would have become much stronger.

Using casts of the vascular system at 906 adult rabbits prepared since 1960 in our laboratory, the ramifications of the aortic arch were surveyed. During these investigations, three individuals in which the left common carotid artery was defective, were accidentally found. Such examples have not been reported previously for rabbits or human subjects. An attempt was therefore made to elucidate the carotid system in each of the three individuals and to assess this kind of abnormality.

\section{Materials and methods}

All vascular casts and acryl-injected 
specimens preserved in formalin were prepared by the acryl plastic injection method (Taniguchi and Ohta et al. 1952, 1955). The plastic was injected via the left ventricle of the heart into the vascular system of the whole body. Two examples (\#1 and \#2) in which the left common carotid artery was found to be defective, occurred in the vascular casts, and the other example (\#3) in the formalin-preserved specimens. The latter specimen was dissected in detail, and a remnant of the left common carotid artery was prepared as serial histological slides by paraffin-embedding and hematoxylin-eosin staining.

\section{Observations}

\section{Ramifications of the aortic arch}

The aortic arch in usual cases gave rise to the brachiocephalic trunk and the left subclavian artery. This trunk divided into the right common carotid artery and the right subclavian artery after giving off the left common cartoid (Type B in man; Adachi 1928) (Fig. 2). However, such a typical pattern was not observed in each of the three cases reported here (Figs. 1 and 3). Their features may be outlined as follows.

The location of the aortic arch $(8 \mathrm{~mm}$ in diameter in $\# 1,6.5 \mathrm{~mm}$ in $\# 2$, and $5.6 \mathrm{~mm}$ in $\# 3$, respectively) ranged from the height of the inferior part of the 7 th cervical vertebral body to the superior part of the 1 st thoracic vertebral body. The brachiocephalic trunk $(4.5 \mathrm{~mm}$ in diameter in $\# 1,3.8 \mathrm{~mm}$ in $\# 2$, and $3.5 \mathrm{~mm}$ in $\# 3$, respectively) arose cranially from the superior wall of the arch, and the left subclavian artery $(2.9 \mathrm{~mm}$ in diameter in \#1, $2.5 \mathrm{~mm}$ in \#2, and $2.2 \mathrm{~mm}$ in \#3 respectively) arose cranially and leftward about $7.5 \mathrm{~mm}$ distal to the origin of the brachiocephalic trunk. This trunk, about $8 \mathrm{~mm}$ distal to this origin, divided cranially into the right common carotid artery $(3.9 \mathrm{~mm}$ in diameter in $\# 1,2.5 \mathrm{~mm}$ in $\# 2$, and $2.9 \mathrm{~mm}$ in \#3, respectively) and rightward the right subclavian artery (3.5 $\mathrm{mm}$ in diameter in $\# 1,2.0 \mathrm{~mm}$ in $\# 2$, and $2.2 \mathrm{~mm}$ in \#3, respectively). Branches did not arise from the arch, or from the brachiocephalic trunk (Figs. 1 and 3 ). In \#3, a fine cord $(0.8 \mathrm{~mm}$ in outer diameter $)$ left the left wall of the origin of the brachiocephalic trunk and passed cranially with the left vagus nerve after running obliquely in front of the trachea leftward and cranially (Fig. 4). In cross sections of the cord at the height of the 4th cervical vertebra, the inner surface surrounding a narrow lumen (40 $\mu \mathrm{m}$ in diameter) appeared to have strong undulations due to the proliferation of endothelial cells and elastic fibers in the internal elastic membrane (Fig. 6a). These features were similar to those of the internal carotid artery of the adult cat which normally degenerates and disappears at birth (Fig. 6b).

II. Right common carotid artery and its branches

Although the right common carotid artery in each of the three examples was normal in its morphology, its diameter (3.9 $\mathrm{mm}$ in \#1,2.5 $\mathrm{mm}$ in \#2, and $2.9 \mathrm{~mm}$ in $\# 3$, respectively) was rather thicker than that in the usual case as shown in Table 1. The right common carotid passed cranially with the right vagus nerve, lateral to the trachea, up to the inferior end of the larynx at the height of the 1 st cervical vertebra, where it divided into the external and internal carotid arteries. At this bifurcation, the carotid body was seen (Figs. 8 and 9). The right external carotid passed cranially posterior to the greater cornu of the hyoid bone and across the medial to the hypoglossal nerve and the digastricus muscle. The right external carotid gave rise to the linguofacial trunk anteriorly inferomedial to the styloglossus muscle in $\# 1$ and $\# 3$. This 
Table 1. Diameters of principal arteries $(\mathrm{mm})$.

\begin{tabular}{|c|c|c|c|c|c|c|c|c|}
\hline \multirow{2}{*}{ Examples } & \multicolumn{2}{|c|}{$\# 1$} & \multicolumn{2}{|c|}{$\# 2$} & \multicolumn{2}{|c|}{$\# 3$} & \multicolumn{2}{|c|}{ Normal } \\
\hline & Right & Left & Right & Left & Right & Left & Right & Left \\
\hline Common carotid & 3.9 & & 2.5 & & 2.9 & & 2.1 & 2.1 \\
\hline External carotid & 2.7 & & 2.4 & & 2.3 & & 2.0 & 2.0 \\
\hline Internal carotid & 1.4 & & 1.2 & 0.5 & 0.9 & 0.7 & 1.1 & 1.1 \\
\hline Lingual & 2.4 & 2.2 & 1.7 & 1.6 & 2.1 & 1.8 & 1.3 & 1.2 \\
\hline Facial & 1.5 & 1.5 & 1.5 & 1.2 & 1.5 & 1.4 & 1.4 & 1.5 \\
\hline Superficial temporal & 2.1 & 2.0 & 1.6 & 1.4 & 1.4 & 1.2 & 1.7 & 1.6 \\
\hline Maxilliary & 2.2 & 1.8 & 1.6 & 1.5 & 1.4 & 1.2 & 1.9 & 1.8 \\
\hline
\end{tabular}

artery in \#3 curved and continued laterally, after giving off the occipital artery posteriorly, between the digastricus and the styloglossus muscles, where it divided into the maxillary and the superficial temporal arteries (Fig. 1). The occipital artery in \#1 arose from the right vertebral artery and in \#2 from the internal carotid. The right external carotid in \#2 divided into the maxillary and the superficial temporal inferomedial to the styloglossus. Both these arteries passed laterally between these muscles. The lingual and the facial arteries in \#2 arose from the maxillary artery. The diameters of these arteries are listed in Table 1. The external carotid and the lingual arteries of the right side were rather thick. The lingual artery passed anteromedially, after giving off the lingual deep artery, lateral to the hyoid body, up to a position cranial to the origin of the hyoglossus muscle. A branch of the lingual artery anastomosed strongly with a similar branch of the opposite lingual without diminishing its diameter (Figs. 7 and 8).

III. Left common carotid artery and its branches
The left common carotid artery, appearing as a fine cord, arose from the brachiocephalic trunk as mentioned above and passed cranially with the left vagus nerve up to the 3rd cervical vertebra, where it became a fine vessel with a narrow lumen. Then, gradually increasing its diameter, it passed cranially and divided into the internal and external carotid, after giving off the superior laryngeal artery at the same height as the right fellow (120 $\mu \mathrm{m}$ in diameter at this height) (Figs. 7, 8, 10 and 11). At this bifurcation, the carotid body was seen. The ramifications of the left carotid in \#1 and \#2 were similar to those of the right fellow. The occipital artery in \#3 arose from the internal carotid. The left lingual was similar to the right one in its diameter, course and ramifications, and anastomosed strongly with the right fellow without diminishing its diameter (Figs. 7 and 8). The left internal carotid $(1.1 \mathrm{~mm}$ in diameter in $\# 1)$ was thinner than the usual vessel. This artery in $\# 2$ and \#3 became diminished in diameter after giving off the occipital $(0.2 \mathrm{~mm}$ in diameter in $\# 1$, and $0.3 \mathrm{~mm}$ in $\# 3$, respectively), but increased its diameter (0.6 $\mathrm{mm}$ in $\# 2$, and $0.7 \mathrm{~mm}$ in $\# 3$, respectively) 
in the cranial cavity.

IV. Blood supply to the left half of the head

The left half of the head was supplied by the strong anastomoses mentioned above between the lingual arteries of both sides (Figs. 7 and 8) due to the lack of the left common carotid. Accordingly, some of the branches in the left area of the head were similar to those of the right lingual artery.

\section{Discussion}

Adachi (1928) and others in man, and Suzuki (1982) and Tanuma et al. (1982) in the Japanese monkey have described the lack of the common carotid artery, respectively. However, both the internal and external carotid arteries in these reported cases arose directly from the aortic arch, the brachiocephalic trunk or the left subclavian artery. Different from these findings, the unilateral common carotid artery in the present three examples was entirely lacking, and the structures of that side were supplied by ramifications of the external carotid of the opposite, normal side. It was important and interesting that the left common carotid itself existed as a remnant consisting of a fine cord of connective tissue, and the distal end continued as a fine vessel. The ramifications of the right carotid in \#1 were similar to Type IA of Okuda et al. (1987) and Yaso (1988), those in \#2 to Type III of Baldwin (1919), and those in \#3 to Type IV of Baldwin and Type ID of Okuda et al. and Yaso.

As observed in the present examples, when only one blood supply route to the unilateral side was defective, this area could be supplied by certain ramifications of the opposite, external carotid in the normal aspect. It was found that the lingual artery, being a thick and proximal branch of the external carotid, became an important vessel as a bilaterally communicating route since this artery in usual cases gave off a thick branch communicating with a similar branch of the opposite fellow. Such a vessel was observed as the hyoid branch located anterior to the hyoid body, and communicated with the opposite fellow in usual cases. Similar descriptions have been given for the cat, goat, and rabbit by Okano (1960) and for the rat by Shimizu (1986) in their work on the lingual artery. In particular, the hyoid branch was always stout in the rabbit. In the present three examples, the hyoid branch was extremely well-developed. Prior to closure of the proximal end of the left common carotid, the well-developed hyoid branch will contribute to supply the left half of the head. In relation to this, the closure of the internal carotid of the cat (Fig. 6b) in disproportion to the development of the ophthalmic artery as well as the closing aspect of Botallo's duct in the rabbit (Ito 1952) resemble the interior aspect of the fine cord in the present three examples (Fig. 6a).

In each of the examples, the right common carotid was not influenced, only the left one. In usual cases in the rabbit, only two branches, namely, the brachiocephalic trunk and the left subclavian artery, arose from the aortic arch. This trunk trifurcated into the right subclavian, the right and left common carotid arteries. This arrangement corresponds to Type B of man (about 10\%). Although the origin of the left common carotid is always located on the right side of the median line, this vessel is directed to the left half of the head. In addition, the left common carotid in most cases of the rabbit is somewhat thinner than that of the right one. The thinness and unusual division of the left common carotid may thus make it liable to damage during the embryological period. 


\section{References}

1) Adachi, B.: Das Arteriensystem der Japaner. Bd I, 22-57, Maruzen Co., Kyoto, 1928.

2) Baldwin, F.M.: Variations in the carotid arteries of the rabbit. Anat. Rec., 16: 309-315, 1919.

3) Ito, S.: Closure of Botallo's duct in newborn infant. J. Tokyo Med. Coll., 10: 1-10, 1952 (in Japanese).

4) Okano, H.: Cubical anatomy of several ducts and vessels by injection method of acrylic resin. VII. On the ramification of A. lingualis and its fine distribution in some mammals. Okajimas Folia Anat. Jpn., 34: 233-297, 1960.

5) Okuda, H., K. Yaso, M. Matsumoto and Y. Ohta: Ramification aspects of the external carotid artery of the rabbit. Acta Anat. Nippon. (Kaibo. Z.) (Abstract), 62: 474, 1987 (in Japanese).

6) Shimizu, T.: The lingual artery of the rat.
Okajimas Folia Anat. Jpn., 63: 47-66, 1986.

7) Suzuki, M.: On the arrangement of the branches arising from the aortic arch in the Japanese monkey. Acta Anat. Nippon., 57: 105-119, 1982 (in Japanese).

8) Taniguchi, Y., Y. Ohta and S. Tajiri: New improved method for injection of acrylic resin. Okajimas Folia Anat. Jpn., 24: 259-267, 1952.

9) Taniguchi, Y., Y. Ohta, S. Tajiri, H. Okano and H. Hanai: Supplement to new improved method for injection of acrylic resin. Okajimas Folia Anat. Jpn., 27: 401-406, 1955.

10) Tanuma, K., M. Suzuki and F. Kikkawa: Two cases of abnormal origins of the left external and internal carotid arteries in the Japanese monkey. Okajimas Folia Anat. Jpn., 58: 1231-1240, 1982.

11) Yaso, K.: Ramification aspects of the external carotid artery of the rabbit. Jpn. J. Oral Biol., 30: 144-155, 1988 (in Japanese). 


\title{
Explanation of Figures
}

\author{
aa : Aortic arch \\ bc : Brachiocephalic trunk \\ ec : External carotid artery \\ f : Facial artery \\ ic : Internal carotid artery \\ 1 : Lingual artery \\ lc : Left common carotid artery
}

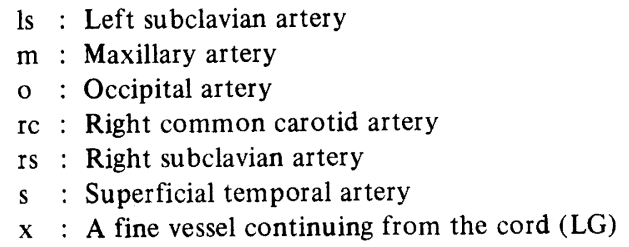

\section{Plate I}

Figs. 1a and 1b. Whole arterial system of the head in $\# 1$, \#2, and \#3 (Fig. 1a) and the respective schema (Fig. 1b). (x 0.5) 
Plate I

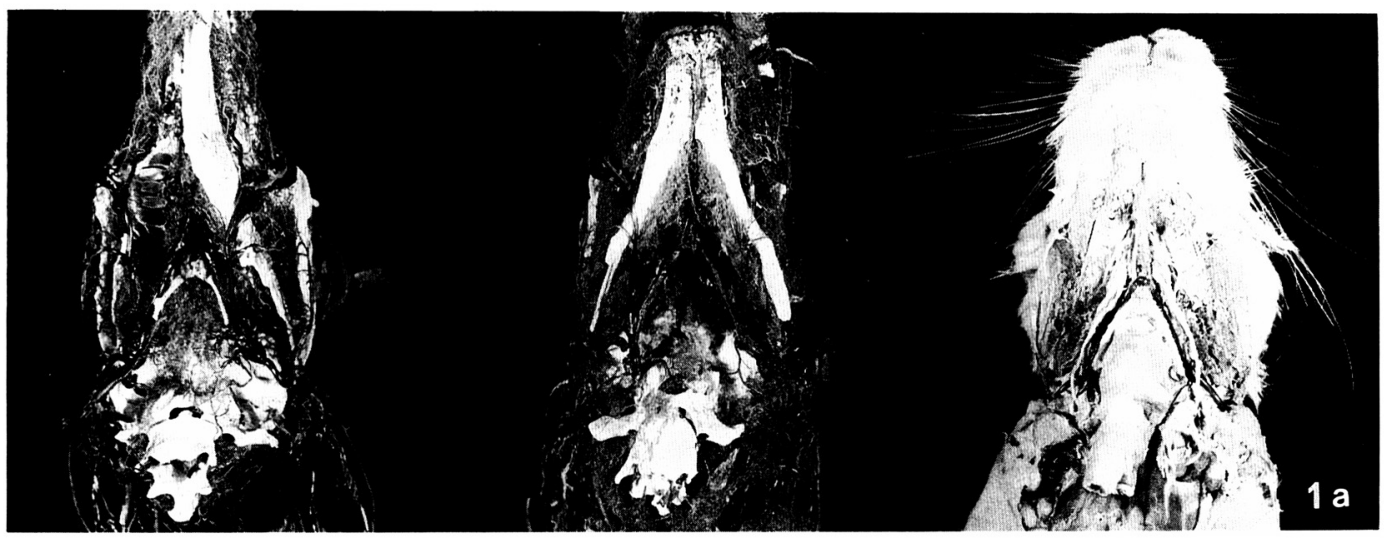

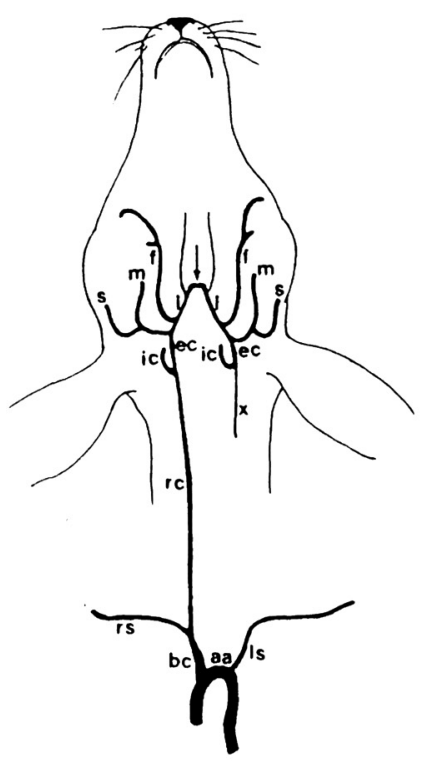

\#1

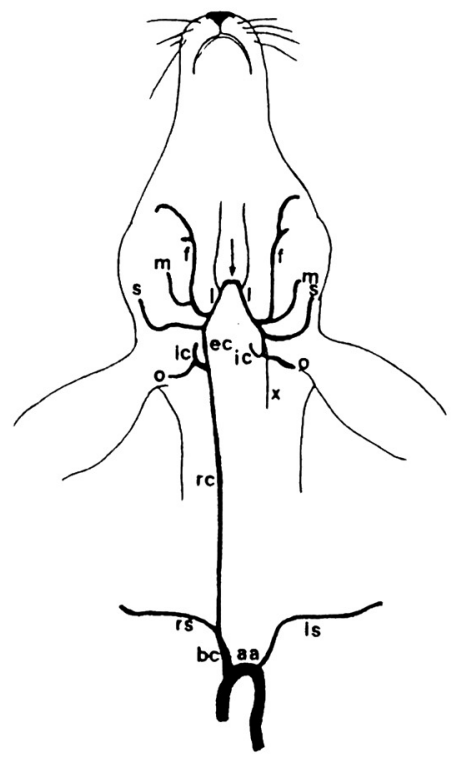

\#2

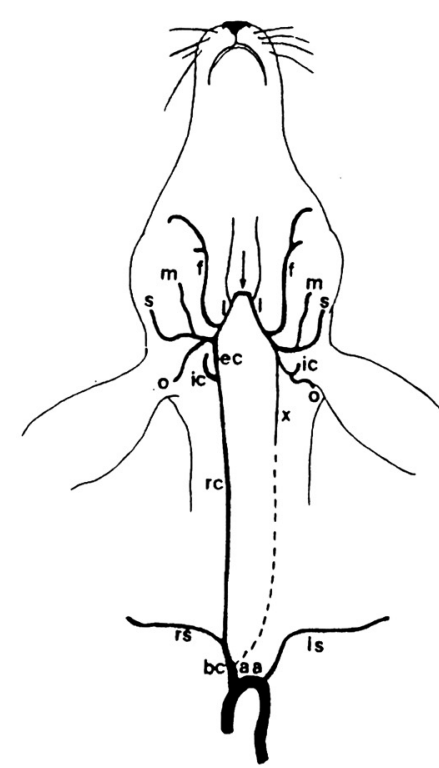

\# 3 


\section{Plate II}

Fig. 2. Normal pattern of branches of the aortic arch. $(\times 1.7)$

Fig. 3. The left common carotid artery $(1 \mathrm{c})$ in $\# 1$ is not observed. $(\times 2)$

Fig. 4. In $\# 3$, a fine cord (LG) is seen instead of the left common carotid artery. $\Delta:$ Left and right vagus nerves. $(\times 1.5)$

Fig. 5. Ramifications of the external carotid arteries in the normal pattern. $(x 1.6)$

An anastomosis $(\downarrow)$ between the bilateral hyoid branches of the lingual arteries is observed in front of the hyoid body $(*)$.

Fig. 6a. Cross section of the cord at the height of C4 in \#3 (shown in Fig. 4). ( $\times 220$ )

6b. Cross section of the degenerated internal carotid artery of an adult cat. ( $\times 220)$

In both figures, proliferations of endothelial cells $\left(\gamma_{0}\right)$ and thickness of the internal elastic membrane $(\rightarrow)$ are observed. 

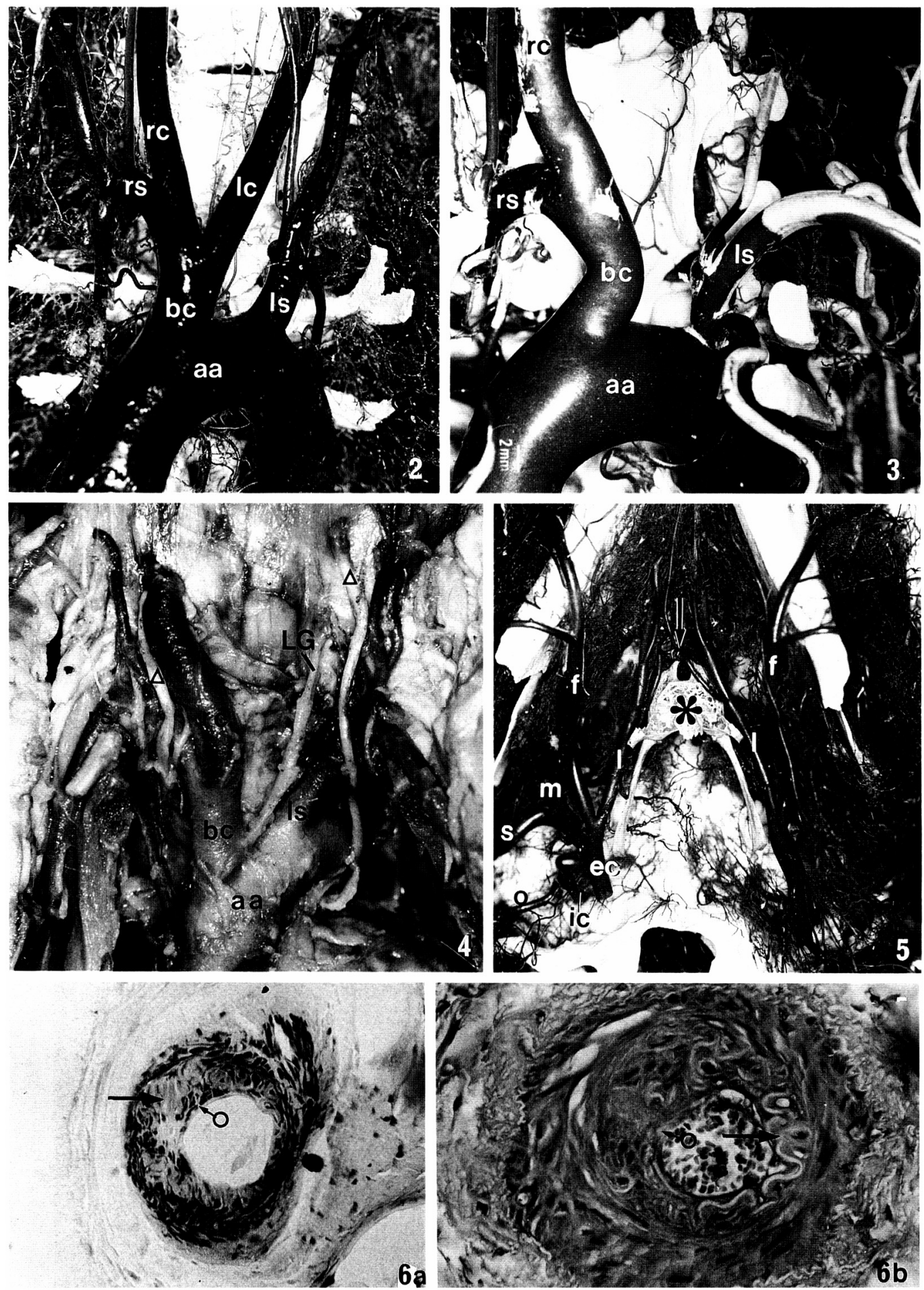


\section{Plate III}

Figs. 7 and 8 . Whole views of $\# 1$ and $\# 3(\times 1.5, \times 1.5$, respectively).

Figs. 9, 10 and 11. Lateral views of the right side of $\# 3$, the left side of $\# 3$, and the left side of $\# 1$. ( $\times 1.5$, $\times 1.5, \times 2$, respectively).

The bilateral lingual arteries are thicker than those of usual cases (Fig. 5) and as well the anastomosis $(\downarrow)$ between them in front of the hyoid body $(*)$. The right common carotid artery is rather thick, but the left one is observed as a fine vessel $(x)$. o: Left carotid body. HG: Hypoglossal nerve. $\triangle$ : Right and left vagus nerves. $\oplus$ : Right carotid body. 

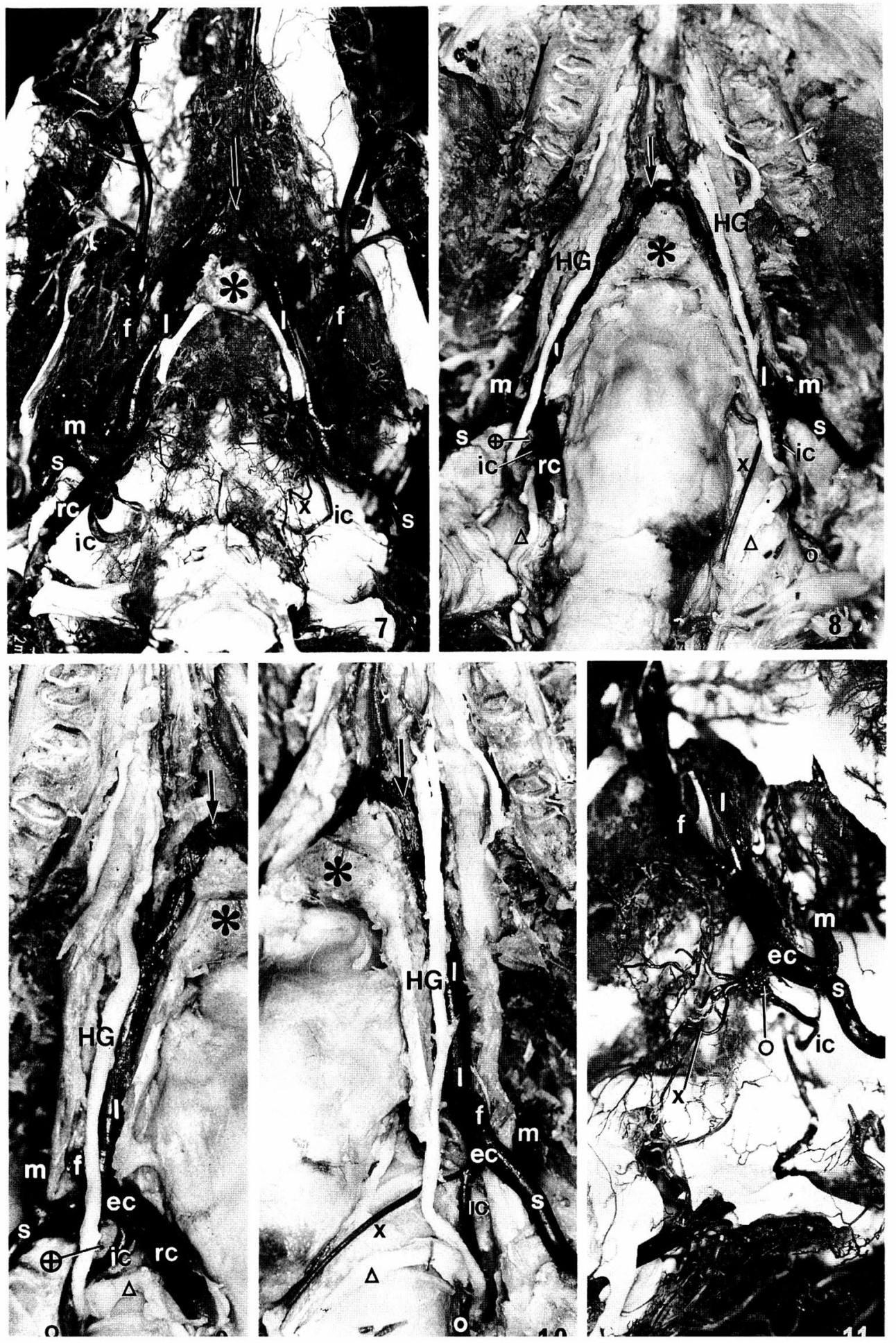